\title{
Discrete symmetries and nonlocal reductions
}

\author{
Metin Gürses ${ }^{a}$, Aslı Pekcan ${ }^{b, *}$, Kostyantyn Zheltukhin ${ }^{c}$ \\ a Department of Mathematics, Faculty of Science, Bilkent University, 06800 Ankara, Turkey \\ b Department of Mathematics, Faculty of Science, Hacettepe University, 06800 Ankara, Turkey \\ c Department of Mathematics, Faculty of Science, Middle East Technical University, 06800 Ankara, Turkey
}

\section{A R T I C L E I N F O}

\section{Article history:}

Received 8 July 2019

Received in revised form 14 October 2019

Accepted 15 October 2019

Available online 21 October 2019

Communicated by F. Porcelli

\section{Keywords:}

Integrable systems

Scale symmetries

Discrete symmetries

Nonlocal reductions

\begin{abstract}
A B S T R A C T
We show that nonlocal reductions of systems of integrable nonlinear partial differential equations are the special discrete symmetry transformations.
\end{abstract}

(C) 2019 Elsevier B.V. All rights reserved.

\section{Introduction}

Nonlocal reductions of systems of integrable nonlinear partial differential equations which were invented first by Ablowitz and Musslimani [1-3], attracted many researchers in the field. Ablowitz and Musslimani have first constructed nonlocal reduction for nonlinear Schrödinger (NLS) system of equations and obtained nonlocal nonlinear Schrödinger (nNLS) equation [1], [2]. They showed that nNLS equation is integrable, i.e., it admits a Lax pair, and found soliton solutions by the use of the inverse scattering method. Ablowitz and Musslimani have later extended their nonlocal reductions, corresponding to space reflection, time reflection, and space-time reflection to modified Korteweg-de Vries (mKdV) system, sine-Gordon (SG) system, Davey-Stewartson (DS) system, and so on. After Ablowitz and Musslimani's works there is a huge interest in obtaining nonlocal reductions of systems of integrable equations and finding interesting wave solutions of these systems. Specific examples are nonlocal NLS equation [1-14], nonlocal mKdV equation [2-4], [13], [15-18], nonlocal SG equation [2-4], [19], nonlocal DS equation [3], [20-24], nonlocal Fordy-Kulish equations [13], [25], nonlocal $N$-wave systems [3], [26], nonlocal vector NLS equations [27-30], nonlocal $(2+1)$-dimensional negative AKNS systems [31], and nonlocal coupled Hirota-Iwao mKdV systems [32]. See [33] for the discussion of superposition of nonlocal

\footnotetext{
* Corresponding author.

E-mail addresses: gurses@fen.bilkent.edu.tr (M. Gürses), aslipekcan@hacettepe.edu.tr (A. Pekcan), zheltukh@metu.edu.tr (K. Zheltukhin).
}

integrable equations, and [34] for the nonlocal reductions of the integrable equations of hydrodynamic type. The connection between local and nonlocal reductions is given in [35], [36]. In all these works the soliton solutions and their properties were investigated by using the inverse scattering method, by the Hirota bilinear method, and by the Darboux transformations.

In the last decade we observe that even as the number of systems of integrable nonlinear differential equations possessing nonlocal reductions is increasing, the origin of nonlocal reductions remains mysterious. Recently, Valchev [37] generalizing the reduction group of Mikhailov [38], (see also [6], [26], [29] for the application of Mikhailov's reduction group) transforming also the independent variables obtained the nonlocal reductions of Ablowitz and Musslimani. In this work we address to this problem. We show that those systems possessing nonlocal reductions admit discrete symmetry transformations which leave the systems invariant. A special case of discrete symmetry transformations turns out to be the nonlocal reductions of the same systems. We show this fact for NLS, mKdV, SG, DS, coupled NLS-derivative NLS, loop soliton systems, hydrodynamic type systems, and Fordy-Kulish equations, and derive all possible nonlocal reductions from the discrete symmetry transformations of these systems.

\section{Reductions}

Let the dynamical variables $q^{i}(t, x)$ and $r^{i}(t, x)(i=1,2, \cdots, N)$, in $(1+1)$-dimensions, satisfy the following system of integrable evolution equations 
$q_{t}^{i}=F^{i}\left(q^{j}, r^{j}, q_{x}^{j}, r_{x}^{j}, q_{x x}^{j}, r_{x x}^{j}, \cdots\right), \quad i, j=1,2, \cdots, N$,

$r_{t}^{i}=G^{i}\left(q^{j}, r^{j}, q_{x}^{j}, r_{x}^{j}, q_{x x}^{j}, r_{x x}^{j}, \cdots\right), \quad i, j=1,2, \cdots, N$,

where $F^{i}$ and $G^{i}(i=1,2, \cdots, N)$ are functions of the dynamical variables $q^{i}(t, x), r^{i}(t, x)$, and their partial derivatives with respect to $x$. The above system of equations is integrable, so it has a Lax pair and a recursion operator $\mathcal{R}$. Some of these equations admit local and nonlocal reductions. Let us assume that the above system of equations (1) and (2) admits the following reductions.

(a) Local reductions:

The local reductions are given by

$r^{i}(t, x)=\kappa_{1} q^{i}(t, x), \quad i=1,2, \cdots, N$,

and

$r^{i}(t, x)=\kappa_{2} \bar{q}^{i}(t, x), \quad i=1,2, \cdots, N$,

where $\kappa_{1}$ and $\kappa_{2}$ are real constants. Throughout this paper a bar over a letter is defined as

1) for a complex number $q=\alpha+i \beta, \bar{q}=\alpha-i \beta, i^{2}=-1$,

2) for a pseudo-complex number $q=\alpha+i \beta, \bar{q}=\alpha-i \beta, i^{2}=1$.

If a reduction is consistent the system of equations (1) and (2) is reduced to a system for $q^{i}$,

$q_{t}^{i}=\tilde{F}^{i}\left(q^{j}, q_{x}^{j}, q_{x x}^{j}, \cdots\right), \quad i, j=1,2, \cdots, N$

for the reduction (3), and

$q_{t}^{i}=\tilde{F}^{i}\left(q^{j}, \bar{q}^{j}, q_{x}^{j}, \bar{q}_{x}^{j}, q_{x x}^{j}, \bar{q}_{x x}^{j}, \cdots\right), \quad i, j=1,2, \cdots, N$

for the reduction (4), where $\tilde{F}=\left.F\right|_{r^{i}=\kappa_{1} q^{i}}$ or $\tilde{F}=\left.F\right|_{r^{i}=\kappa_{2} \bar{q}^{i}}$, respectively.

(b) Nonlocal reductions:

Recently, Ablowitz and Musslimani introduced new type of reductions [1-3]

$r^{i}(t, x)=\tau_{1} q^{i}\left(\varepsilon_{1} t, \varepsilon_{2} x\right)=\tau_{1} q_{\varepsilon}^{i}$,

and

$r^{i}(t, x)=\tau_{2} \bar{q}^{i}\left(\varepsilon_{1} t, \varepsilon_{2} x\right)=\tau_{2} \bar{q}_{\varepsilon}^{i}$,

for $i=1,2, \cdots, N$. Here $\tau_{1}$ and $\tau_{2}$ are real constants and $\varepsilon_{1}^{2}=\varepsilon_{2}^{2}=$ 1.

When $\left(\varepsilon_{1}, \varepsilon_{2}\right)=(-1,1),(1,-1),(-1,-1)$, the above constraints reduce the system (1) and (2) to nonlocal space reflection symmetric (S-symmetric), time reflection symmetric (Tsymmetric), or space-time reflection symmetric (ST-symmetric) differential equations.

Since the reductions are done consistently the reduced systems of equations are also integrable. This means that the reduced systems admit recursion operators and Lax pairs. We can obtain $\mathrm{N}$-soliton solutions of the reduced systems by the inverse scattering method [1-3], [10], [11], [14], [17], [19], [27], by the Darboux transformation [9], [16], [18], [22], [23], and by the Hirota bilinear method [7], [13], [15], [21], [31-33].

\section{Discrete symmetries}

In this section we will show that nonlocal reductions arise from scaling symmetries of integrable system of equations. A scaling symmetry of a system of differential equations is the scale transformation which leaves these equations invariant. Scaling symmetries group is a subgroup of the symmetry groups of differential equations [39] and discrete symmetries are special cases of the scaling symmetries [40].

(a) NLS system: This system is given by

$$
\begin{aligned}
& a q_{t}=-\frac{1}{2} q_{x x}+q^{2} r, \\
& a r_{t}=\frac{1}{2} r_{x x}-q r^{2},
\end{aligned}
$$

where $a$ is any constant. This constant is the imaginary unit for the original NLS system but we change it by redefining the $t$ variable. We search for a symmetry transformation such that the NLS system is left invariant. In general we choose the symmetry transformation as

$T_{1}:(q(t, x), r(t, x)) \rightarrow\left(q^{\prime}\left(t^{\prime}, x^{\prime}\right), r^{\prime}\left(t^{\prime}, x^{\prime}\right)\right)$

where primed system satisfies also the NLS system, i.e.,

$$
\begin{aligned}
& a q_{t^{\prime}}^{\prime}=-\frac{1}{2} q_{x^{\prime} x^{\prime}}^{\prime}+\left(q^{\prime}\right)^{2} r^{\prime}, \\
& a r_{t^{\prime}}^{\prime}=\frac{1}{2} r_{x^{\prime} x^{\prime}}^{\prime}-q^{\prime}\left(r^{\prime}\right)^{2} .
\end{aligned}
$$

We shall consider the real and complex dynamical systems separately.

(1) For the real case the symmetry transformation that we are interested in is the scale transformations

$t^{\prime}=\beta t, \quad x^{\prime}=\alpha x, \quad q^{\prime}=\gamma_{1} q+\delta_{1} r, \quad r^{\prime}=\gamma_{2} r+\delta_{2} q$,

where $\alpha, \beta, \gamma_{1}, \gamma_{2}, \delta_{1}$, and $\delta_{2}$ are real constants. We have two possible cases:

(1.a) First type of real scale symmetry transformation is

$$
t^{\prime}=-\alpha^{2} t, \quad x^{\prime}=\alpha x, \quad q^{\prime}=\delta_{1} r, \quad r^{\prime}=\frac{1}{\delta_{1} \alpha^{2}} q,
$$

where $\alpha$ and $\delta_{1}$ are arbitrary constants.

(1.b) Second type of real scale symmetry transformation is

$$
t^{\prime}=\alpha^{2} t, \quad x^{\prime}=\alpha x, \quad q^{\prime}=\gamma_{1} q, \quad r^{\prime}=\frac{1}{\gamma_{1} \alpha^{2}} r
$$

where $\alpha$ and $\gamma_{1}$ are arbitrary constants. These two parameter scale transformations map solutions to solutions of the NLS system.

From the above scale symmetry transformation we can obtain discrete symmetry transformations by letting $\alpha=\epsilon= \pm 1$. In particular the first type produces a discrete symmetry transformation if $\alpha=\epsilon$ and $\delta_{1}=k$ then

$q(t, x)=k r^{\prime}(-t, \epsilon x), \quad r(t, x)=k q^{\prime}(-t, \epsilon x)$,

where $\epsilon^{2}=k^{2}=1$. A special discrete symmetry transformation is obtained when we take $q^{\prime}=q$ and $r^{\prime}=r$. Under this special discrete symmetry the transformations in (16) reduce to the well-known nonlocal reductions $r(t, x)=k q(-t, x)$ and $r(t, x)=$ $k q(-t,-x)$ [3], [4], [6], [10], [13], [14]

(2) For the complex dynamical systems the scale symmetry transformation

$T_{2}:(\bar{q}(t, x), \bar{r}(t, x)) \rightarrow\left(q^{\prime}\left(t^{\prime}, x^{\prime}\right), r^{\prime}\left(t^{\prime}, x^{\prime}\right)\right)$

takes the following form

$t^{\prime}=\beta t, \quad x^{\prime}=\alpha x, \quad q^{\prime}=\gamma_{1} \bar{q}+\delta_{1} \bar{r}, \quad r^{\prime}=\gamma_{2} \bar{r}+\delta_{2} \bar{q}$,

where $\alpha, \beta, \gamma_{1}, \gamma_{2}, \delta_{1}$, and $\delta_{2}$ are real constants. We have two possible cases: 
(2.a) First type of complex scale symmetry transformation is

$t^{\prime}=\beta t, \quad x^{\prime}=\alpha x, \quad q^{\prime}=\delta_{1} \bar{r}, \quad r^{\prime}=\delta_{2} \bar{q}$,

with

$\bar{a} \beta=-a \alpha^{2}, \quad \delta_{1} \delta_{2} \alpha^{2}=1$.

(2.b) Second type of complex scale symmetry transformation is

$t^{\prime}=\beta t, \quad x^{\prime}=\alpha x, \quad q^{\prime}=\gamma_{1} \bar{q}, \quad r^{\prime}=\gamma_{2} \bar{r}$,

with

$$
\bar{a} \beta=a \alpha^{2}, \quad \gamma_{1} \gamma_{2} \alpha^{2}=1 .
$$

These two parameter scale transformations map also solutions to solutions of the NLS system. From these scale symmetry transformations we obtain discrete symmetry transformation by letting $\alpha=\epsilon_{1}= \pm 1, \beta=\epsilon_{2}= \pm 1, \gamma_{1}=\gamma_{2}=k= \pm 1$. In particular the first type produces a discrete symmetry transformation of the form

$q(t, x)=k \bar{r}^{\prime}\left(\epsilon_{2} t, \epsilon_{1} x\right), \quad r(t, x)=k \bar{q}^{\prime}\left(\epsilon_{2} t, \epsilon_{1} x\right)$,

where $\epsilon_{1}^{2}=\epsilon_{2}^{2}=k^{2}=1$ and $\bar{a} \epsilon_{2}=-a$ which follows from (19). A special discrete symmetry transformation is obtained when we take $q^{\prime}=q$ and $r^{\prime}=r$. Under this special discrete symmetry the transformations in (22) reduce to the well-known nonlocal reductions $r(t, x)=k \bar{q}(-t, x)$ with $\bar{a}=-a, r(t, x)=k \bar{q}(t,-x)$ with $\bar{a}=a$, and $r(t, x)=k \bar{q}(-t,-x)$ with $\bar{a}=-a$ [1], [2], [4-9], [11-14].

The examples that we consider in the rest of the paper share similar real and complex scale symmetry transformations and the associated discrete symmetry transformations. Since we are interested in nonlocal reductions of the integrable systems of equations we will present only the first type real and complex discrete transformations and the corresponding nonlocal reductions.

(b) MKdV system: This system is given by

$$
\begin{aligned}
& a q_{t}=-\frac{1}{4} q_{x x x}+\frac{3}{2} q r q_{x}, \\
& a r_{t}=-\frac{1}{4} r_{x x x}+\frac{3}{2} q r r_{x} .
\end{aligned}
$$

We will write the discrete symmetry transformations directly. We have two different cases: Let $(q, r)$ and $\left(q^{\prime}, r^{\prime}\right)$ satisfy the mKdV system of equations (23) and (24).

For the real case we have

$q(t, x)=k r^{\prime}\left(\epsilon_{2} t, \epsilon_{1} x\right), r(t, x)=k q^{\prime}\left(\epsilon_{2} t, \epsilon_{1} x\right)$,

where $k^{2}=1$ and $\epsilon_{1} \epsilon_{2}=1$. When we take $q^{\prime}=q$ and $r^{\prime}=r$ we obtain the nonlocal reduction $r(t, x)=k q(-t,-x)$ [2-4], [13], [15-17].

For the complex case we have

$q(t, x)=k \bar{r}^{\prime}\left(\epsilon_{2} t, \epsilon_{1} x\right), \quad r(t, x)=k \bar{q}^{\prime}\left(\epsilon_{2} t, \epsilon_{1} x\right)$,

where $\bar{a} \epsilon_{1} \epsilon_{2}=a$ and $k^{2}=1$. These special discrete transformations produce different nonlocal reductions when $q^{\prime}=q$ and $r^{\prime}=r$ with different values of $\epsilon_{1}= \pm 1$ and $\epsilon_{2}= \pm 1 ; r(t, x)=k \bar{q}(-t, x)$ with $\bar{a}=-a, r(t, x)=k \bar{q}(t,-x)$ with $\bar{a}=-a$, and $r(t, x)=k \bar{q}(-t,-x)$ with $\bar{a}=a$ [2-4], [13], [15], [18].

(c) SG system: This system is given by

$$
\begin{aligned}
& q_{x t}+2 s q=0, \\
& r_{x t}+2 s r=0, \\
& s_{x}+(q r)_{t}=0,
\end{aligned}
$$

where $q=q(t, x), r=r(t, x)$, and $s=s(t, x)$. We have the following two discrete symmetry transformations. For the real case,

$$
\begin{aligned}
& q(t, x)=k r^{\prime}\left(\epsilon_{2} t, \epsilon_{1} x\right), \quad r(t, x)=k q^{\prime}\left(\epsilon_{2} t, \epsilon_{1} x\right), \\
& s(t, x)=s^{\prime}\left(\epsilon_{2} t, \epsilon_{1} x\right),
\end{aligned}
$$

where $\epsilon_{1}=\epsilon_{2}= \pm 1$ and $k^{2}=1$. If we take $q^{\prime}=q$ and $r^{\prime}=r$ these special discrete transformations produce the nonlocal reductions: $r(t, x)=k q(-t, x), r(t, x)=k q(t,-x)$, and $r(t, x)=k q(-t,-x)$ [2-4], [19].

For the complex case,

$q(t, x)=k \bar{r}^{\prime}\left(\epsilon_{2} t, \epsilon_{1} x\right), \quad r(t, x)=k \bar{q}^{\prime}\left(\epsilon_{2} t, \epsilon_{1} x\right)$,

$s(t, x)=\bar{s}^{\prime}\left(\epsilon_{2} t, \epsilon_{1} x\right)$,

where $\epsilon_{1}=\epsilon_{2}= \pm 1$ and $k^{2}=1$. When $q^{\prime}=q$ and $r^{\prime}=r$ these special discrete transformations produce the nonlocal reductions: $r(t, x)=k \bar{q}(-t, x), r(t, x)=k \bar{q}(t,-x)$, and $r(t, x)=k \bar{q}(-t,-x)[4]$.

(d) DS system: This system is given by

$$
\begin{aligned}
a q_{t}+\frac{1}{2}\left[\gamma^{2} q_{x x}+q_{y y}\right]+q^{2} r & =\phi q, \\
-a r_{t}+\frac{1}{2}\left[\gamma^{2} r_{x x}+r_{y y}\right]+r^{2} q & =\phi r, \\
\phi_{x x}-\gamma^{2} \phi_{y y} & =2(q r)_{x x},
\end{aligned}
$$

where $q=q(t, x, y), r=r(t, x, y), \phi=\phi(t, x, y), \gamma^{2}= \pm 1$, and $a$ is a constant. We have the following discrete symmetry transformations. For the real case,

$$
\begin{aligned}
& q(t, x, y)=k r^{\prime}\left(\epsilon_{1} t, \epsilon_{2} x, \epsilon_{3} y\right), \\
& r(t, x, y)=k q^{\prime}\left(\epsilon_{1} t, \epsilon_{2} x, \epsilon_{3} y\right), \\
& \phi(t, x, y)=\phi^{\prime}\left(\epsilon_{1} t, \epsilon_{2} x, \epsilon_{3} y\right),
\end{aligned}
$$

where $\epsilon_{1}=-1$ and $k^{2}=1$. These special discrete transformations produce the nonlocal reductions when $q^{\prime}=q$ and $r^{\prime}=r$ with different values of $\epsilon_{1}=-1, \epsilon_{2}= \pm 1, \epsilon_{3}= \pm 1 ; r(t, x, y)=k q(-t, x, y)$, $r(t, x, y)=k q(-t,-x, y), r(t, x, y)=k q(-t, x,-y)$, and $r(t, x, y)=$ $k q(-t,-x,-y)[3]$.

For the complex case,

$$
\begin{aligned}
& q(t, x, y)=k \bar{r}^{\prime}\left(\epsilon_{1} t, \epsilon_{2} x, \epsilon_{3} y\right), \\
& r(t, x, y)=k \bar{q}^{\prime}\left(\epsilon_{1} t, \epsilon_{2} x, \epsilon_{3} y\right), \\
& \phi(t, x, y)=\bar{\phi}^{\prime}\left(\epsilon_{1} t, \epsilon_{2} x, \epsilon_{3} y\right),
\end{aligned}
$$

where $k^{2}=1, \epsilon_{1}^{2}=\epsilon_{2}^{2}=\epsilon_{3}^{2}=1$, and $\bar{a} \epsilon_{1}=-a$. We observe that these discrete transformations produce many different nonlocal reductions when $q^{\prime}=q, r^{\prime}=r$, and $\phi^{\prime}=\phi$ with different values of $\epsilon_{1}= \pm 1, \epsilon_{2}= \pm 1$, and $\epsilon_{3}= \pm 1 ; r(t, x, y)=k \bar{q}(-t, x, y)$, $r(t, x, y)=k \bar{q}(-t,-x, y), \quad r(t, x, y)=k \bar{q}(-t, x,-y), \quad r(t, x, y)=$ $k \bar{q}(-t,-x,-y)$ with $\bar{a}=a ; r(t, x, y)=k \bar{q}(t,-x, y), r(t, x, y)=$ $k \bar{q}(t, x,-y), r(t, x, y)=k \bar{q}(t,-x,-y)$ with $\bar{a}=-a$ [3], [20-24].

(e) Coupled NLS-derivative NLS system: This system [41] is given by

$$
\begin{aligned}
& a q_{t}=i q_{x x}+\alpha\left(r q^{2}\right)_{x}+i \beta r q^{2}, \\
& a r_{t}=-i r_{x x}+\alpha\left(r q^{2}\right)_{x}-i \beta r^{2} q,
\end{aligned}
$$

where $\alpha, \beta \in \mathbb{R}$, and $a$ is any constant. We have the following discrete symmetry transformations. For the real case,

$q(t, x)=k r^{\prime}\left(\epsilon_{2} t, \epsilon_{1} x\right), \quad r(t, x)=k q^{\prime}\left(\epsilon_{2} t, \epsilon_{1} x\right)$, 
where $\epsilon_{1}=\epsilon_{2}=-1$ and $k^{2}=1$. When $q^{\prime}=q$ and $r^{\prime}=r$, these discrete transformations produce the nonlocal reduction $r(t, x)=$ $k q(-t,-x)[3]$.

For the complex case,

$q(t, x)=k \bar{r}^{\prime}\left(\epsilon_{2} t, \epsilon_{1} x\right), \quad r(t, x)=k \bar{q}^{\prime}\left(\epsilon_{2} t, \epsilon_{1} x\right)$,

where $\epsilon_{1}=1, \bar{a} \epsilon_{2}=a$, and $k^{2}=1$. From these discrete transformations we have different nonlocal reductions when $q^{\prime}=q$ and $r^{\prime}=r$ with different values of $\epsilon_{1}= \pm 1$ and $\epsilon_{2}= \pm 1 ; r(t, x)=$ $k \bar{q}(-t, x)$ with $\bar{a}=a, r(t, x)=k \bar{q}(t,-x)$ with $\bar{a}=-a$, and $r(t, x)=$ $k \bar{q}(-t,-x)$ with $\bar{a}=-a$.

(f) Loop-soliton system: This system [41], [42] is given by

$$
\begin{aligned}
& a q_{t}+\frac{\partial^{2}}{\partial x^{2}}\left[\frac{q_{x}}{(1-r q)^{3 / 2}}\right]=0 \\
& a r_{t}+\frac{\partial^{2}}{\partial x^{2}}\left[\frac{r_{x}}{(1-r q)^{3 / 2}}\right]=0
\end{aligned}
$$

We have the following discrete symmetry transformations.

For the real case,

$q(t, x)=k r^{\prime}\left(\epsilon_{2} t, \epsilon_{1} x\right), \quad r(t, x)=k q^{\prime}\left(\epsilon_{2} t, \epsilon_{1} x\right)$,

where $\epsilon_{1}=\epsilon_{2}=-1$ and $k^{2}=1$. When $q^{\prime}=q$ and $r^{\prime}=r$, these discrete transformations produce the nonlocal reduction $r(t, x)=$ $k q(-t,-x)[3]$.

For the complex case,

$q(t, x)=k \bar{r}^{\prime}\left(\epsilon_{2} t, \epsilon_{1} x\right), \quad r(t, x)=k \bar{q}^{\prime}\left(\epsilon_{2} t, \epsilon_{1} x\right)$,

where $\bar{a} \epsilon_{1} \epsilon_{2}=a$, and $k^{2}=1$. These discrete transformations produce different nonlocal reductions when $q^{\prime}=q$ and $r^{\prime}=r$ with different values of $\epsilon_{1}= \pm 1$ and $\epsilon_{2}= \pm 1 ; r(t, x)=k \bar{q}(-t, x)$ with $\bar{a}=-a, r(t, x)=k \bar{q}(t,-x)$ with $\bar{a}=-a$, and $r(t, x)=k \bar{q}(-t,-x)$ with $\bar{a}=a$.

\section{(g) Hydrodynamic type of systems: Shallow water waves}

Recently we studied the reductions in equations of hydrodynamic type [34] and obtained several examples of nonlocal version of these equations. An example of equations of hydrodynamic type is the shallow water waves system [43]

$$
\begin{aligned}
& a q_{t}=(q+r) q_{x}+q r_{x}, \\
& a r_{t}=(q+r) r_{x}+r q_{x} .
\end{aligned}
$$

Here $a$ is a nonzero constant. The discrete transformations which leave this system invariant are following. For the real case,

$r(t, x)=k q^{\prime}\left(\epsilon_{2} t, \epsilon_{1} x\right), \quad q(t, x)=k r^{\prime}\left(\epsilon_{2} t, \epsilon_{1} x\right)$,

where $k=\epsilon_{1} \epsilon_{2}$. For the complex case

$r(t, x)=k \bar{q}^{\prime}\left(\epsilon_{2} t, \epsilon_{1} x\right), \quad q(t, x)=k, \bar{r}^{\prime}\left(\epsilon_{2} t, \epsilon_{1} x\right)$,

where $\bar{a} k \epsilon_{1} \epsilon_{2}=a$. In both cases $k^{2}=\epsilon_{1}^{2}=\epsilon_{2}^{2}=1$ [34].

If we let $q^{\prime}=q$ and $r^{\prime}=r$ we get the special discrete symmetry transformations which lead to the local and nonlocal reductions. When $q$ and $r$ are real variables we have $r(t, x)=k q\left(\epsilon_{2} t, \epsilon_{1} x\right)$ then the reduced equation is

$a q_{t}(t, x)=\left(q(t, x)+k q\left(\epsilon_{2} t, \epsilon_{1} x\right)\right) q_{x}(t, x)+k q(t, x) q_{x}\left(\epsilon_{2} t, \epsilon_{1} x\right)$,

provided that $k=\epsilon_{1} \epsilon_{2}$ and $a$ is real.

When $q$ and $r$ are complex variables we have $r(t, x)=k \bar{q}\left(\epsilon_{2} t . \epsilon_{1} x\right)$ then the reduced equation is $a q_{t}(t, x)=\left(q(t, x)+k \bar{q}\left(\epsilon_{2} t, \epsilon_{1} x\right)\right) q_{x}(t, x)+k q(t, x) \bar{q}_{x}\left(\epsilon_{2} t, \epsilon_{1} x\right)$,

provided that $\bar{a} k \epsilon_{1} \epsilon_{2}=a[34]$.

\section{(h) Fordy-Kulish equations}

Let $q^{\alpha}(t, x)$ and $r^{\alpha}(t, x)$ be the complex dynamical variables where $\alpha=1,2, \cdots, N$, then the Fordy-Kulish (FK) integrable system is given by [44]

$$
\begin{aligned}
a q_{t}^{\alpha} & =q_{x x}^{\alpha}+R_{\beta \gamma-\delta}^{\alpha} q^{\beta} q^{\gamma} r^{\delta}, \\
-a r_{t}^{\alpha} & =r_{x x}^{\alpha}+R_{-\beta-\gamma \delta}^{-\alpha} r^{\beta} r^{\gamma} q^{\delta},
\end{aligned}
$$

where $R^{\alpha}{ }_{\beta \gamma-\delta}, R^{-\alpha}-\beta-\gamma \delta$ are the curvature tensors of a Hermitian symmetric space with

$$
\left(R_{\beta \gamma-\delta}^{\alpha}\right)^{\star}=R_{-\beta-\gamma \delta}^{-\alpha},
$$

and $a$ is a complex number. Here we use the summation convention, i.e., the repeated indices are summed up from 1 to $N$. These equations are known as the FK system which is integrable in the sense that they are obtained from the zero curvature condition of a connection defined on a Hermitian symmetric space. The FK equations (55) and (56) are invariant under the discrete transformations

$r^{\alpha}(t, x)=k \bar{q}^{\prime \alpha}\left(\epsilon_{1} t, \epsilon_{2} x\right), \quad q^{\alpha}(t, x)=k \bar{r}^{\prime \alpha}\left(\epsilon_{1} t, \epsilon_{2} x\right)$,

where $k^{2}=\epsilon_{1}^{2}=\epsilon_{2}^{2}=1$ and $\bar{a} \epsilon_{1}=-a$. If we let $r^{\prime \alpha}=r^{\alpha}$ and $q^{\prime \alpha}=$ $q^{\alpha}$ we obtain the special discrete symmetry transformations and hence the nonlocal reductions $r^{\alpha}(t, x)=k \bar{q}^{\alpha}\left(\epsilon_{1} t, \epsilon_{2} x\right)$ [25]. Then the reduced nonlocal FK equations are

$a q_{t}^{\alpha}(t, x)=q_{x x}^{\alpha}(t, x)+k R_{\beta \gamma-\delta}^{\alpha} q^{\beta}(t, x) q^{\gamma}(t, x) \bar{q}^{\delta}\left(\epsilon_{1} t, \epsilon_{2} x\right)$.

\section{Conclusion}

In this work we showed that the discrete symmetries of systems of integrable equations are important in finding the nonlocal reductions. For this reason we started first with the scale symmetry transformations of real and complex dynamical systems. Discrete symmetry transformations are special cases of the scale transformations. There are two different types, called as the first and second types, of discrete symmetry transformations both for real and complex dynamical variables. Using this fact we can find all discrete symmetry transformations of the system of equations. Among these discrete symmetry transformations the first types are the origins of the nonlocal reductions of these systems. We showed that a special discrete symmetry transformation of the first type produces all the well-known nonlocal reductions.

\section{Declaration of competing interest}

The authors declare that they have no known competing financial interests or personal relationships that could have appeared to influence the work reported in this paper.

\section{References}

[1] M.J. Ablowitz, Z.H. Musslimani, Integrable nonlocal nonlinear Schrödinger equation, Phys. Rev. Lett. 110 (2013) 064105.

[2] M.J. Ablowitz, Z.H. Musslimani, Inverse scattering transform for the integrable nonlocal nonlinear Schrödinger equation, Nonlinearity 29 (2016) 915-946.

[3] M.J. Ablowitz, Z.H. Musslimani, Integrable nonlocal nonlinear equations, Stud. Appl. Math. 139 (1) (2016) 7-59.

[4] K. Chen, X. Deng, S. Lou, D. Zhang, Solutions of local and nonlocal equations reduced from the AKNS hierarchy, Stud. Appl. Math. 141 (2018) 113-141. 
[5] B.F. Feng, X.D. Luo, M.J. Ablowitz, Z.H. Musslimani, General soliton solution to a nonlocal nonlinear Schrödinger equation with zero and nonzero boundary conditions, Nonlinearity 31 (12) (2018) 5385-5409.

[6] V.S. Gerdjikov, A. Saxena, Complete integrability of nonlocal nonlinear Schrödinger equation, J. Math. Phys. 58 (1) (2017) 013502.

[7] M. Gürses, A. Pekcan, Nonlocal nonlinear Schrödinger equations and their soliton solutions, J. Math. Phys. 59 (2018) 051501.

[8] X. Huang, L. Ling, Soliton solutions for the nonlocal nonlinear Schrödinger equation, Eur. Phys. J. Plus 131 (2016) 148.

[9] M. Li, T. Xu, Dark and antidark soliton interactions in the nonlocal nonlinear Schrödinger equation with the self-induced parity-time-symmetric potential, Phys. Rev. E 91 (2015) 033202.

[10] M.J. Ablowitz, B.F. Feng, X.D. Luo, Z.H. Musslimani, Inverse scattering transform for the nonlocal reverse space-time nonlinear Schrödinger equation, Theor Math. Phys. 196 (3) (2018) 1241-1267.

[11] X.Y. Wen, Z. Yan, Y. Yang, Dynamics of higher-order rational solitons for the nonlocal nonlinear Schrödinger equation with the self-induced parity-timesymmetric potential, Chaos 26 (2015) 063123.

[12] A. Khare, A. Saxena, Periodic and hyperbolic soliton solutions of a number of nonlocal nonlinear equations, J. Math. Phys. 56 (2015) 032104.

[13] M. Gürses, A. Pekcan, Integrable nonlocal reductions, in: V.G. Kac, P.J. Olver, P. Winternitz, T. Ozer (Eds.), Symmetries, Differential Equations and Applications SDEA-III, Istanbul, Turkey, August 2017, in: Springer Proceedings in Mathematics and Statistics, vol. 266, 2018, pp. 27-52.

[14] J. Yang, General N-solitons and their dynamics in several nonlocal nonlinear Schrödinger equations, Phys. Lett. A 383 (4) (2019) 328-337.

[15] M. Gürses, A. Pekcan, Nonlocal nonlinear modified KdV equations and their soliton solutions, Commun. Nonlinear Sci. Numer. Simul. 67 (2019) 427-448.

[16] J.L. Ji, Z.N. Zhu, On a nonlocal modified Korteweg-de Vries equation: integrability, Darboux transformation and soliton solutions, Commun. Nonlinear Sci. Numer. Simul. 42 (2017) 699-708.

[17] J.L. Ji, Z.N. Zhu, Soliton solutions of an integrable nonlocal modified Kortewegde Vries equation through inverse scattering transform, J. Math. Anal. Appl. 453 (2017) 973-984.

[18] L.Y. Ma, S.F. Shen, Z.N. Zhu, Soliton solution and gauge equivalence for an integrable nonlocal complex modified Korteweg-de Vries equation, J. Math. Phys. 58 (2017) 103501

[19] M.J. Ablowitz, B.F. Feng, X.D. Luo, Z.H. Musslimani, Reverse space-time nonlocal sine-Gordon/sinh-Gordon equations with nonzero boundary conditions, Stud Appl. Math. 141 (2018) 267-307.

[20] A.S. Fokas, Integrable multidimensional versions of the nonlocal Schrödinger equation, Nonlinearity 29 (2016) 319.

[21] J. Rao, Y. Zhang, A.S. Fokas, J. He, Rogue waves of the nonlocal DaveyStewartson I equation, Nonlinearity 31 (2018) 4090-4107.

[22] T. Xu, M. Li, Y. Huang, Y. Chen, C. Yu, Nonsingular localized wave solutions for the nonlocal Davey-Stewartson I equation with zero background, Mod. Phys. Lett. B 31 (35) (2017) 1750338.
[23] Z.-X. Zhou, Darboux transformations global explicit solutions for nonlocal Davey-Stewartson I equation, Stud. Appl. Math. 141 (2) (2018) 186-204.

[24] Y. Zhang, Y. Liu, Breather and lump solutions for nonlocal Davey-Stewartson II equation, Nonlinear Dyn. 96 (2019) 107-113.

[25] M. Gürses, Nonlocal Fordy-Kulish equations on symmetric spaces, Phys. Lett. A 381 (2017) 1791.

[26] V.S. Gerdjikov, G.G. Grahovski, R.I. Ivanov, The N-wave equations with PT symmetry, Theor. Math. Phys. 188 (3) (2016) 1305

[27] D. Sinha, P.K. Ghosh, Integrable nonlocal vector nonlinear Schrödinger equation with self-induced parity-time symmetric potential, Phys. Lett. A 381 (2017) 124.

[28] Z. Yan, Integrable PT-symmetric local and nonlocal vector nonlinear Schrödinger equations: a unified two parameter model, Appl. Math. Lett. 47 (2015) 61.

[29] V.S. Gerdjikov, G.G. Grahovski, R.I. Ivanov, On integrable wave interactions and Lax pairs on symmetric spaces, Wave Motion 71 (2017) 53.

[30] V.S. Gerdjikov, On nonlocal models of Kulish-Sklyanin type and generalized Fourier transforms, Stud. Comput. Intell. 681 (2017) 37.

[31] M. Gürses, A. Pekcan, $(2+1)$-dimensional local and nonlocal reductions of the negative AKNS system: soliton solutions, Commun. Nonlinear Sci. Numer. Simul. 71 (2019) 161-173.

[32] A. Pekcan, Nonlocal coupled HI-MKdV systems, Commun. Nonlinear Sci. Numer Simul. 72 (2019) 493-515.

[33] M. Gürses, A. Pekcan, Superposition of NLS and mKdV equations, Appl. Math. Lett. 98 (2019) 157-163.

[34] M. Gürses, A. Pekcan, K. Zheltukhin, Nonlocal hydrodynamic type of equations, arXiv:1906.08475 [nlin.SI].

[35] V. Caudrelier, Interplay between the inverse scattering method and Fokas's unified transform with an application, Stud. Appl. Math. 140 (2017) 3.

[36] B. Yang, J. Yang, Transformations between nonlocal and local integrable equations, Stud. Appl. Math. 140 (2017) 178.

[37] T. Valchev, On Mikhailov's reduction group, Phys. Lett. A 379 (2015) 1877-1880.

[38] A.V. Mikhailov, The reduction problem and the inverse scattering method, Physica D 3 (1981) 73-117.

[39] P.J. Olver, Applications of Lie Groups to Differential Equations, second ed., Graduate Texts in Mathematics, vol. 107, Springer Verlag, 1986.

[40] P.E. Hydon, Symmetry Methods for Differential Equations: A Beginner's Guide, Cambridge University Press, 2000.

[41] M.J. Ablowitz, P.A. Clarkson, Solitons, Nonlinear Evolution Equations and Inverse Scattering, Cambridge University Press, Cambridge, 1991.

[42] K. Konno, A. Jeffrey, The loop soliton, in: L. Debnath (Ed.), Advances in Nonlinear Waves, vol. I, Pitman, London, 1984, pp. 162-183.

[43] M. Gürses, K. Zheltukhin, Recursion operators of some equations of hydrodynamic type, J. Math. Phys. 42 (3) (2001) 1309-1325.

[44] A.P. Fordy, P.P. Kulish, Nonlinear Schrödinger equations and simple Lie algebras, Commun. Math. Phys. 89 (1983) 427-443. 